\title{
APPLICATIONS OF WEIGHTED LAGUERRE TRANSPLANTATION THEOREMS*
}

\author{
GEORGE GASPER ${ }^{\dagger}$ AND WALTER TREBELS T $^{\ddagger}$
}

To Richard Askey on his 65th birthday

\begin{abstract}
As applications of the weighted transplantation theorems in Stempak and Trebels [16] we consider (i) the characterization of one-dimensional Hermite multipliers via Laguerre multipliers, (ii) extension theorems for Laguerre multipliers in the spirit of Coifman and Weiss [3, Theorem 6.5], and (iii) necessary conditions for Laguerre multipliers via backward differences.
\end{abstract}

1. Introduction and Notations. The purpose of this paper is to apply the weighted version [16] of Kanjin's transplantation theorem [12] for Laguerre expansions in the following three instances:

(i) We characterize the one-dimensional Hermite multipliers on $L^{p}, 1<p<\infty$, via corresponding Laguerre multipliers. As a corollary of the results for Laguerre multipliers we obtain a sufficient criterion of Hörmander type for Hermite multipliers which is slightly better than that in Thangavelu [17, p. 91].

(ii) Coifman and Weiss [3, Theorem 6.5] related radial Fourier multipliers on $L^{p}\left(\mathbf{R}^{n}\right)$ with those on $L^{p}\left(\mathbf{R}^{n+2}\right)$ via transference methods. We deduce an (improved) analog for Laguerre multipliers which is in the nature of best possible.

(iii) Necessary conditions for Laguerre multipliers are derived via backward differences. Though these are not sharp they nevertheless give the impression that necessary conditions of Kalner̆-type (see [11] for the Jacobi series case, see [8] for the Laguerre series case) viewed till now as isolated in the framework of known necessary [5] (sufficient [16]) criteria arise from backward differences.

The paper is of programmatic character: We can show in the integer case that transplantation theorems reflect the structure of corresponding multiplier spaces, but fail to extend the result indicated in (ii) to fractional differences at the moment. Also it is evident that a full range transplantation theorem with general power weights is needed (i.e. the analog of Muckenhoupt's result [14, Theorem 1.6] for Jacobi series).

To become more precise let us introduce some notation. Let $L_{n}^{\alpha}(x), \alpha>-1, n \in$ $\mathbf{N}_{0}$, be the classical Laguerre polynomials (see [18, p. 100]),

$$
R_{n}^{\alpha}(x)=L_{n}^{\alpha}(x) / L_{n}^{\alpha}(0), \quad L_{n}^{\alpha}(0)=A_{n}^{\alpha}=\left(\begin{array}{c}
n+\alpha \\
n
\end{array}\right)=\frac{\Gamma(n+\alpha+1)}{\Gamma(n+1) \Gamma(\alpha+1)},
$$

be the normalized ones. Introducing a forward difference operator $\Delta^{\lambda}$ and a backward one $\nabla^{\lambda}$ by (whenever the sums exist)

$$
\Delta^{\lambda} c_{k}=\sum_{j=0}^{\infty} A_{j}^{-\lambda-1} c_{k+j}, \quad \nabla^{\lambda} c_{k}=\sum_{j=0}^{k} A_{j}^{-\lambda-1} c_{k-j},
$$

*Received Oct 10, 1998, revised March 8, 1999.

†Department of Mathematics, Northwestern University, Evanston, IL 60208, USA (george@ math.nwu.edu). The work of this author was supported in part by the National Science Foundation under grant DMS-9401452.

$\ddagger$ Fachbereich Mathematik, TU Darmstadt, Schloßgartenstr. 7, D-64289 Darmstadt, Germany (trebels@mathematik.tu-darmstadt.de). Part of this work was done while the author was visiting the Department of Mathematics at Northwestern University, which he wishes to thank for its hospitality. 
we call into mind two identities $[4,6.15(4), 10.12(39)]$, essential for the following.

$$
\begin{gathered}
\Delta^{\lambda} R_{k}^{\alpha}(x)=C_{\alpha, \lambda} x^{\lambda} R_{k}^{\alpha+\lambda}(x), \quad x>0, \lambda>-(\alpha+1 / 2) / 2, \\
\nabla^{\lambda} L_{k}^{\alpha+\lambda}(x)=L_{k}^{\alpha}(x), \quad x>0, \lambda \in \mathbf{R} .
\end{gathered}
$$

Using (2) and an interchange of the summation order yields for finite sequences $\left\{a_{k}\right\}$

$$
\sum_{k=0}^{\infty}\left(\Delta^{\lambda} a_{k}\right) L_{k}^{\alpha+\lambda}(x)=\sum_{k=0}^{\infty} a_{k} L_{k}^{\alpha}(x)
$$

from which we can conclude

$$
\left\|\sum_{k=0}^{\infty} a_{k} L_{k}^{\alpha}\right\|_{L_{w(\alpha)}^{p}} \leq C\left\|\sum_{k=0}^{\infty}(k+1)^{\lambda}\left(\Delta^{\lambda} a_{k}\right) L_{k}^{\alpha}\right\|_{L_{w(\alpha)}^{p}}, \quad \lambda>0,1 \leq p<\infty .
$$

For, by $[2,(3.30)]$, we have

$$
\begin{gathered}
\left\|\sum_{k=0}^{\infty} a_{k} L_{k}^{\alpha}\right\|_{L_{w(\alpha)}^{p}} \\
\leq C\left(\int_{0}^{\infty}\left|\int_{0}^{1}(1-t)^{\lambda-1} t^{\alpha} \sum_{k} \frac{\Gamma(k+\alpha+\lambda+1)}{\Gamma(k+\alpha+1)}\left(\Delta^{\lambda} a_{k}\right) L_{k}^{\alpha}(x t) d t e^{-x / 2}\right|^{p} x^{\alpha} d x\right)^{1 / p} \\
\leq C \int_{0}^{1}(1-t)^{\lambda-1} t^{\alpha-(\alpha+1) / p}\left(\int_{0}^{\infty}\left|\sum_{k} \frac{\Gamma(k+\alpha+\lambda+1)}{\Gamma(k+\alpha+1)}\left(\Delta^{\lambda} a_{k}\right) L_{k}^{\alpha}(y) e^{-y / 2}\right|^{p} y^{\alpha} d y\right)^{1 / p} d t
\end{gathered}
$$

when using the integral Minkowski inequality. Observing that $\{\Gamma(k+\alpha+\lambda+1) /(\Gamma(k+$ $\left.\left.\alpha+1)(k+1)^{\lambda}\right)\right\} \in M_{\alpha ; \alpha}^{p}$ for all $p$, the assertion (4) follows. Generic positive constants that are independent of the functions (and sequences) will be denoted by $C$. To a function $f \in L_{w(\gamma)}^{p}$, where

$$
L_{w(\gamma)}^{p}=\left\{f:\|f\|_{L_{w(\gamma)}^{p}}=\left(\int_{0}^{\infty}\left|f(x) e^{-x / 2}\right|^{p} x^{\gamma} d x\right)^{1 / p}<\infty\right\}, \quad 1 \leq p<\infty,
$$

one can associate its formal Laguerre series

$$
f(x) \sim(\Gamma(\alpha+1))^{-1} \sum_{k=0}^{\infty} \hat{f}_{\alpha}(k) L_{k}^{\alpha}(x), \quad \hat{f}_{\alpha}(n)=\int_{0}^{\infty} f(x) R_{n}^{\alpha}(x) x^{\alpha} e^{-x} d x .
$$

A scalar-valued sequence $m=\left\{m_{k}\right\}_{k \in \mathrm{N}_{0}}$ is called a (bounded) multiplier on $L_{w(\gamma)}^{p}$, notation $m \in M_{\alpha ; \gamma}^{p}$, if

$$
\left\|\sum_{k=0}^{\infty} m_{k} a_{k} L_{k}^{\alpha}\right\|_{L_{w(\gamma)}^{p}} \leq C\left\|\sum_{k=0}^{\infty} a_{k} L_{k}^{\alpha}\right\|_{L_{w(\gamma)}^{p}}
$$

for all polynomials $f=(\Gamma(\alpha+1))^{-1} \sum a_{k} L_{k}^{\alpha}$ (which are dense in $L_{w(\gamma)}^{p}$ for appropriate $\gamma$ - see Poiani [15, Theorem 2] ); the smallest constant $C$ for which this holds is called the multiplier norm $\|m\|_{M_{\alpha_{i} \gamma}^{p}}$. We observe the duality property $\left(1 / p+1 / p^{\prime}=1\right)$

$$
M_{\alpha ; \gamma}^{p}=M_{\alpha ; \alpha p^{\prime}-\gamma p^{\prime} / p}^{p^{\prime}}, \quad-1<\gamma<p(\alpha+1)-1, \quad 1<p<\infty
$$


and, therefore, can restrict ourselves to the case $p \leq 2$ in the following.

The weighted transplantation theorem [16, Corollary 4.3] we will apply can be formulated as follows

$$
M_{\alpha ; \alpha+\delta}^{p}=M_{\beta ; \beta}^{p}, \quad \beta=\alpha+\frac{2 \delta}{2-p}, \quad 1<p<2, \quad \alpha, \beta>-1,
$$

provided $\beta$ satisfies the condition

$$
\begin{cases}(2 \beta+2)\left(\frac{1}{p}-\frac{1}{2}\right)<1 & \text { if } \quad \alpha, \beta \geq 0 \\ -1<\beta<0 & \text { if } \quad \beta<0 \text { and } \alpha>\beta \\ \beta<\frac{\alpha p}{2-p}+\frac{2(p-1)}{2-p} & \text { if } \quad \alpha<0 \text { and } \alpha<\beta\end{cases}
$$

This relation already indicates the essential role played by transplantation theorems in the examination of the structure of multiplier spaces. Let us now turn to our first application, the characterization of Hermite multipliers.

2. Hermite multipliers. The Hermite polynomials are given by [18, p. 106]

$$
H_{n}(x)=(-1)^{n} e^{x^{2}}\left(\frac{d}{d x}\right)^{n} e^{-x^{2}}, \quad x \in \mathbf{R}, n \in \mathbf{N}_{0} .
$$

For $1 \leq p<\infty$ define the Lebesgue spaces

$$
L_{w(H ; \gamma)}^{p}=\left\{f:\|f\|_{L_{w(H ; \gamma)}^{p}}=\left(\int_{-\infty}^{\infty}\left|f(x) e^{-x^{2} / 2}\right|^{p}|x|^{\gamma} d x\right)^{1 / p}<\infty\right\}, \quad \gamma>-1 ;
$$

$\gamma>-1$ is assumed to ensure that $H_{k} \in L_{w(H ; \gamma)}^{p}$ for all $k \in \mathbf{N}_{0}$. Define in the canonical way Hermite coefficients $\hat{f}_{H}(k)$ of $f \in L_{w(H ; \gamma)}^{p}$ by

$$
\hat{f}_{H}(k)=h_{k}^{H} \int_{-\infty}^{\infty} f(t) H_{k}(t) e^{-t^{2}} d t, \quad h_{k}^{H}=\left(\int_{-\infty}^{\infty}\left[H_{k}(x)\right]^{2} e^{-x^{2}} d x\right)^{-1}=\left(\sqrt{\pi} 2^{k} k !\right)^{-1} .
$$

By Hölder's inequality, the $\hat{f}_{H}(k)$ exist if $\gamma<p-1$; for these $\gamma$ associate to $f \in L_{w(H ; \gamma)}^{p}$ its Hermite expansion

$$
f(x) \sim \sum_{k=0}^{\infty} \hat{f}_{H}(k) H_{k}(x) .
$$

We call a scalar-valued sequence $m=\left\{m_{k}\right\}_{k \in N_{0}}$ a Hermite multiplier, $m \in M_{H ; \gamma}^{p}$, if for $T_{m} f \sim \sum m_{k} \hat{f}_{H}(k) H_{k}(x)$ there holds

$$
\left\|T_{m} f\right\|_{L_{w(H ; \gamma)}^{p}} \leq\|m\|_{M_{H ; \gamma}^{p}}\|f\|_{L_{w(H ; \gamma)}^{p}} ;
$$

if $\gamma=0$ write $M_{H ; 0}^{p}=M_{H}^{p}$. Since the polynomials are dense in $L_{w(H ; \gamma)}^{p},-1<\gamma<$ $p-1$, (see [15, Theorem 7]) we restrict ourselves in the following to polynomial $f$. Now observe that $H_{2 n}$ are even, $H_{2 n+1}$ are odd polynomials so that one can uniquely decompose $f \in L_{w(H ; \gamma)}^{p}$ into its even and its odd part,

$$
f_{e}(x)=\frac{1}{2}(f(x)+f(-x)), \quad f_{o}(x)=\frac{1}{2}(f(x)-f(-x)) .
$$


Then

$$
\|f\|_{L_{w(H ; \gamma)}^{p}} \leq\left\|f_{e}\right\|_{L_{w(H ; \gamma)}^{p}}+\left\|f_{o}\right\|_{L_{w(H ; \gamma)}^{p}} \leq 2\|f\|_{L_{w(H ; \gamma)}^{p}}
$$

and for their Hermite coefficients we obtain

$$
\left(f_{e}\right)_{H}(k)=\left\{\begin{array}{ll}
\hat{f}_{H}(k) & , \quad k \text { even } \\
0 & , \quad k \text { odd }
\end{array}, \quad\left(f_{o}\right)_{H}(k)=\left\{\begin{array}{ll}
0 & , k \text { even } \\
\hat{f}_{H}(k) & , k \text { odd }
\end{array} .\right.\right.
$$

From this it is clear (see [7] in the ultraspherical case) that the $M_{H ; \gamma}^{p}$-multiplier norm of $m$ is equivalent to the multiplier norm of $m$ restricted to the subspace of even $L_{w(H ; \gamma)}^{p}$-functions plus the multiplier norm of $m$ restricted to the subspace of odd $L_{w(H ; \gamma)}^{p}$-functions, i.e.,

$$
\|m\|_{M_{H ; \gamma}^{p}} \approx\|m\|_{\left.M_{H ; \gamma}^{p}\right|_{\text {even }}}+\|m\|_{\left.M_{H ; \gamma}^{p}\right|_{\text {odd }}} .
$$

Via quadratic transformations $[18,(5.6 .1)]$ one can reduce the Hermite polynomials to Laguerre polynomials

$$
H_{2 m}(x)=(-1)^{m} 2^{2 m} m ! L_{m}^{-1 / 2}\left(x^{2}\right), \quad H_{2 m+1}(x)=(-1)^{m} 2^{2 m+1} m ! x L_{m}^{1 / 2}\left(x^{2}\right) ;
$$

thus in particular

$$
\begin{gathered}
\sum_{k=0}^{\infty} m_{2 k} \hat{f}_{H}(2 k) H_{2 k}(x)=\sum_{k=0}^{\infty} m_{2 k} a_{k} L_{k}^{-1 / 2}\left(x^{2}\right), \quad a_{k}=(-1)^{k} 2^{2 k} k ! \hat{f}_{H}(2 k), \\
\sum_{k=0}^{\infty} m_{2: k+1} \hat{f}_{H}(2 k+1) H_{2 k+1}(x)=\sum_{k=0}^{\infty} m_{2 k+1} b_{k} x L_{k}^{1 / 2}\left(x^{2}\right), \quad b_{k}=(-1)^{k} 2^{2 k+1} k ! \hat{f}_{H}(2 k+1)
\end{gathered}
$$

Since

$$
\left\|\sum_{k=0}^{\infty} m_{2 k} \hat{f}_{H}(2 k) H_{2 k}\right\|_{L_{w(H ; \gamma)}^{p}}=C\left(\int_{0}^{\infty}\left|\sum_{k=0}^{\infty} m_{2 k} a_{k} L_{k}^{-1 / 2}(t) e^{-t / 2}\right|^{p} t^{(\gamma-1) / 2} d t\right)^{1 / p},
$$

it immediately follows that

$$
\left\|\left\{m_{2 k}\right\}\right\|_{\left.M_{H ; \gamma}^{p}\right|_{\text {even }}} \approx\left\|\left\{m_{2 k}\right\}\right\|_{M_{-1 / 2 ;(\gamma-1) / 2}^{p}}
$$

similarly

$$
\left\|\left\{m_{2 k+1}\right\}\right\|_{\left.M_{H ; \gamma}^{p}\right|_{\text {odd }}} \approx\left\|\left\{m_{2 k+1}\right\}\right\|_{M_{1 / 2 ;(\gamma+p-1) / 2}^{p}}
$$

and, therefore,

$$
\left\|\left\{m_{k}\right\}\right\|_{M_{H ; \gamma}^{p}} \approx\left(\left\|\left\{m_{2 k}\right\}\right\|_{M_{-1 / 2 ;(\gamma-1) / 2}^{p}}+\left\|\left\{m_{2 k+1}\right\}\right\|_{M_{1 / 2 ;(\gamma+p-1) / 2}^{p}}\right) .
$$

If we now apply the transplantation theorem (5) in the case $\gamma=0$, we obtain the announced characterization, see part (a) of the following corollary.

Corollary 2.1 (a) For $1<p<\infty$ there holds

$$
\left\|\left\{m_{k}\right\}\right\|_{M_{H}^{p}} \approx\left(\left\|\left\{m_{2 k}\right\}\right\|_{M_{-1 / 2 ;-1 / 2}^{p}}+\left\|\left\{m_{2 k+1}\right\}\right\|_{M_{-1 / 2 ;-1 / 2}^{p}}\right) .
$$


(b) Defining $\Delta_{2} m_{k}=m_{k}-m_{k+2}$ we have for $1<p<\infty$

$$
\left\|\left\{m_{k}\right\}\right\|_{M_{H}^{p}} \leq C\left(\|m\|_{\ell \infty}+\sup _{N}\left(\sum_{N}^{2 N}(k+1)\left|\Delta_{2} m_{k}\right|^{2}\right)^{1 / 2}\right) .
$$

(c) For $4 / 3<p<4$ the following sufficient condition is true

$$
\left\|\left\{m_{k}\right\}\right\|_{M_{H ; 1-p / 2}^{p}} \leq C\left(\|m\|_{\ell^{\infty}}+\sup _{N}\left(\sum_{N}^{2 N}(k+1)\left|\Delta_{2} m_{k}\right|^{2}\right)^{1 / 2}\right) .
$$

Part (a) and [16, Corollary 4.5] imply (b), (5) for $\gamma=1-p / 2$, duality and [16, Theorem 1.1] give (c) of Corollary 2.1. The two sufficient Hermite multiplier criteria (b) and (c) contain those of Thangavelu [17, Theorem 4.2.1] for one dimension and even improve them slightly.

3. An extension theorem in the spirit of Coifman and Weiss. In $[3$, Theorem 6.5], Coifman and Weiss have shown the following extension result for radial Fourier multipliers:

Denote by $\xi_{(k)}=\left(\xi_{1}, \ldots, \xi_{k}\right), k \in \mathbf{N}$, a vector in the $k$-dimensional Euclidean space $\mathbf{R}^{k}$. If $\left[t^{1-n}\left(t^{n} m(t)\right)^{\prime}\right]_{t=\left|\xi_{(n)}\right|}, t \geq 0$, is a Fourier multiplier on $L^{p}\left(\mathbf{R}^{n}\right)$, then $m\left(\left|\xi_{(n+2)}\right|\right) \in M^{p}\left(\mathbf{R}^{n+2}\right)$ and

$$
\left\|m\left(\left|\xi_{(n+2)}\right|\right)\right\|_{M^{p}\left(\mathbf{R}^{n+2}\right)} \leq C\left\|\left[t^{1-n}\left(t^{n} m(t)\right)^{\prime}\right]_{t=\left|\xi_{(n)}\right|}\right\|_{M^{p}\left(\mathbf{R}^{n}\right)}, \quad 1 \leq p \leq \infty .
$$

While for $p=1$ this result is best possible, it is of course not natural for $p=2$ (recall $M^{2}\left(\mathbf{R}^{k}\right)=L^{\infty}\left(\mathbf{R}^{k}\right)$ ). On account of Zafran's result [19] one cannot directly improve it via interpolation. Here we want to give an analog of the Coifman and Weiss result in the framework of Laguerre multipliers, an analog which is in the nature of best possible, thus indicating what to look for in the Fourier multiplier case. Having established the Laguerre multiplier result, on account of Guy's [10] transplantation theorem, the corresponding result for (modified) Hankel multipliers is obvious, thus a result for radial Fourier multipliers restricted to radial functions.

On account of duality we will restrict ourselves to the case $1 \leq p \leq 2$.

Let us start with the case $1<p<2$; by (3) we have for finite sequences $\left\{a_{k}\right\}_{k \in \mathrm{N}_{0}}$ that

$$
\begin{aligned}
& \left\|\sum_{k=0}^{\infty} m_{k} a_{k} L_{k}^{\alpha}\right\|_{L_{w(\alpha)}^{p}}=\left\|\sum_{k=0}^{\infty} \Delta\left(m_{k} a_{k}\right) L_{k}^{\alpha+1}\right\|_{L_{w(\alpha)}^{p}} \\
& \leq\left\|\sum_{k=0}^{\infty}(k+1)\left(\Delta m_{k}\right) \frac{a_{k}}{k+1} L_{k}^{\alpha+1}\right\|_{L_{w(\alpha)}^{p}}+\left\|\sum_{k=0}^{\infty} m_{k+1}\left(\Delta a_{k}\right) L_{k}^{\alpha+1}\right\|_{L_{w(\alpha)}^{p}} \\
& \leq\left\|\left\{(k+1) \Delta m_{k}\right\}\right\|_{M_{\alpha+1 ; \alpha}^{p}}\left\|\sum_{k=0}^{\infty} \frac{a_{k}}{k+1} L_{k}^{\alpha+1}\right\|_{L_{w(\alpha)}^{p}}+\left\|\left\{m_{k+1}\right\}\right\|_{M_{\alpha+1 ; \alpha}^{p}}\left\|\sum_{k=0}^{\infty} \Delta a_{k} L_{k}^{\alpha+1}\right\|_{L_{w(\alpha)}^{p}} \\
& \leq C\left(\left\|\left\{(k+1) \Delta m_{k}\right\}\right\|_{M_{\alpha+1 ; \alpha}^{p}}+\left\|\left\{m_{k+1}\right\}\right\|_{M_{\alpha+1 ; \alpha}^{p}}\right)\left\|\sum a_{k} L_{k}^{\alpha}\right\|_{L_{w(\alpha)}^{p}},
\end{aligned}
$$

where the first estimate on the right hand side follows from [2, (3.30)] and [1, Corollary $2.2]$ and the second from (3). Hence

$$
\|m\|_{M_{\alpha ; \alpha}^{p}} \leq C\left(\left\|\left\{(k+1) \Delta m_{k}\right\}\right\|_{M_{\alpha+1 ; \alpha}^{p}}+\left\|\left\{m_{k+1}\right\}\right\|_{M_{\alpha+1 ; \alpha}^{p}}\right)
$$


An application of the transplantation result (5) immediately gives then

C'OROLlaRY 3.1 Let $1<p<2$; if $\beta=\alpha-\frac{p}{2-p}>-1$ satisfies $(2 \beta+2)\left(\frac{1}{p}-\frac{1}{2}\right)<1$,

$$
\|m\|_{M_{\alpha ; \alpha}^{p}} \leq C\left(\left\|\left\{(k+1) \Delta m_{k}\right\}\right\|_{M_{\beta ; \beta}^{p}}+\left\|\left\{m_{k+1}\right\}\right\|_{M_{\beta ; \beta}^{p}}\right) .
$$

REMARK 1. Concerning smoothness of the involved multiplier sequences, this result is in accordance with the necessary conditions in [5, II, Corollary 1.3] and the sufficient ones in [16, Corollary 1.2]. In both types of conditions the smoothness of an $M_{\beta ; \beta}^{p}$-multiplier sequence is described by the quantities $(2 \beta+1)\left(\frac{1}{p}-\frac{1}{2}\right)$ and $(2 \beta+2)\left(\frac{1}{p}-\frac{1}{2}\right)$, resp.. Increasing the parameter from $\beta$ to $\alpha, \alpha$ and $\beta$ as in Corollary 3.1 , should require an additional smoothness of 1 in the necessary conditions as well as in the sufficient ones; this being true is at once verified since $2 \alpha\left(\frac{1}{p}-\frac{1}{2}\right)-2 \beta\left(\frac{1}{p}-\frac{1}{2}\right)=1$. Thus the counterexamples showing that the necessary conditions and the sufficient ones just mentioned could not be improved within the setting of $w b v$-spaces can be taken to show that Corollary 3.1 is best possible.

2. It is clear that a full range transplantation theorem with general power weights would remove the restriction on $\beta$ - see also Corollary 3.2 below where the case $p=1$ is discussed and for whose proof no transplantation theorem is needed.

Let us now turn to the case $p=1$; consider again a finite sequence $\left\{a_{k}\right\}$ and assume without loss of generality that $a_{0}=0$. By [18, (5.1.14)] we have for $\alpha>-1$ that

$$
x \sum a_{k} L_{k}^{\alpha+1}=(1+\alpha) \sum a_{k} L_{k}^{\alpha}-\sum(k+1)\left(\Delta a_{k}\right) L_{k+1}^{\alpha}
$$

which implies that

$$
\left\|\sum a_{k} L_{k}^{\alpha+1}\right\|_{L_{w(\alpha+1)}^{1}} \approx\left\|\sum(k+1) \Delta a_{k} L_{k}^{\alpha}\right\|_{L_{w(\alpha)}^{1}} .
$$

The ":-"-direction directly follows by the triangle inequality, (4), and Proposition 3.3 (a) below which discusses the boundedness of the shift operator; the converse inequality is a consequence of the triangle inequality (from below) and the restriction result in [6, Theorem 2.1].

Corollary 3.2 For $\alpha \geq 0$ there holds

$$
\|m\|_{M_{\alpha+1 ; \alpha+1}^{1}} \approx\left\|\left\{m_{k+1}\right\}\right\|_{M_{\alpha ; \alpha}^{1}}+\left\|\left\{(k+1) \Delta m_{k}\right\}\right\|_{M_{\alpha ; \alpha}^{1}} .
$$

REMARK 3. The additional assumption $\alpha \geq 0$ arises from the circumstance that the boundedness of the generalized Laguerre translation, hence of the convolution, has only been proved for these $\alpha$-values - see [9]; but this property is used in the proof of (8) below.

Let us start with the " $\leq$ "-direction. From (7) we have with the aid of (4)

$$
\begin{aligned}
& \left\|\sum m_{k} a_{k} L_{k}^{\alpha+1}\right\|_{L_{w(\alpha+1)}^{1}} \leq C\left\|\sum(k+1)\left(\Delta m_{k} a_{k}\right) L_{k}^{\alpha}\right\|_{L_{w(\alpha)}^{1}} \\
\leq & C\left\|\sum(k+1)\left(\Delta m_{k}\right) a_{k} L_{k}^{\alpha}\right\|_{L_{w(\alpha)}^{1}}+C\left\|\sum m_{k+1}(k+1)\left(\Delta a_{k}\right) L_{k}^{\alpha}\right\|_{L_{w(\alpha)}^{1}} \\
\leq & C\left(\left\|\left\{m_{k+1}\right\}\right\|_{M_{\alpha ; \alpha}^{1}}+\left\|\left\{(k+1) \Delta m_{k}\right\}\right\|_{M_{\alpha ; \alpha}^{1}}\right)\left\|\sum(k+1)\left(\Delta a_{k}\right) L_{k}^{\alpha}\right\|_{L_{w(\alpha)}^{1}} \\
\leq & C\left(\left\|\left\{m_{k+1}\right\}\right\|_{M_{\alpha ; \alpha}^{1}}+\left\|\left\{(k+1) \Delta m_{k}\right\}\right\|_{M_{\alpha ; \alpha}^{1}}^{1}\right)\left\|\sum a_{k} L_{k}^{\alpha+1}\right\|_{L_{w(\alpha+1)}^{1}}
\end{aligned}
$$


by (7). For the converse we note that by [6] we have for $\alpha \geq 0$

$$
\|m\|_{M_{\alpha ; \alpha}^{1}} \approx \sup _{0<r<1}\left\|\sum r^{k} m_{k} L_{k}^{\alpha}\right\|_{L_{w(\alpha)}^{1}}, \quad\|m\|_{M_{\alpha ; \alpha}^{1}} \leq C\|m\|_{M_{\alpha+1 ; \alpha+1}^{1}} .
$$

Thus, up to the proof of the fact that the shift of a multiplier sequence is a bounded operator in the multiplier norm, there remains to estimate uniformly in $r, 0<r<1$,

$$
\begin{aligned}
& \left\|\sum r^{k}(k+1)\left(\Delta m_{k}\right) L_{k}^{\alpha}\right\|_{L_{w(\alpha)}^{1}} \\
\leq & \left\|\sum(k+1)\left(\Delta r^{k} m_{k}\right) L_{k}^{\alpha}\right\|_{L_{w(\alpha)}^{1}}+\left\|\sum m_{k+1}(k+1)\left(\Delta r^{k}\right) L_{k}^{\alpha}\right\|_{L_{w(\alpha)}^{1}} \\
\leq & C\left\|\sum m_{k} r^{k} L_{k}^{\alpha+1}\right\|_{L_{w(\alpha+1)}^{1}}+C\left\|\left\{m_{k+1}\right\}\right\|_{M_{\alpha ; \alpha}^{1}}\left\|\sum(k+1)(1-r) r^{k} L_{k}^{\alpha}\right\|_{L_{w(\alpha)}^{1}} \\
\leq & C\left(\|m\|_{M_{\alpha+1 ; \alpha+1}^{1}}+\left\|\left\{m_{k+1}\right\}\right\|_{M_{\alpha ; \alpha}^{1}}\right)
\end{aligned}
$$

by (7) since, when applying [5, II, Theorem 3.1], it turns out that $\left\{r^{k}\right\}$ as well as $\left\{(k+1)(1-r) r^{k}\right\}$ are Laguerre coefficients of $L_{w(\alpha)}^{1}$-functions, whose $L^{1}$-norms are uniformly bounded in $r$; hence the assertion via (8) and the boundedness of the shift operator which we prove below.

Proposition 3.3 (a) Let $\alpha>-1$ and $1 \leq p \leq 2$. Then

$$
\left\|\sum_{k=0}^{\infty} a_{k} L_{k+1}^{\alpha}\right\|_{L_{w(\alpha)}^{p}} \leq C\left\|\sum_{k=0}^{\infty} a_{k} L_{k}^{\alpha}\right\|_{L_{w(\alpha)}^{p}} .
$$

Also, setting $L_{-1}^{\alpha}(x)=0$, the converse holds:

$$
\left\|\sum_{k=0}^{\infty} a_{k} L_{k-1}^{\alpha}\right\|_{L_{w(\alpha)}^{p}} \leq C\left\|\sum_{k=0}^{\infty} a_{k} L_{k}^{\alpha}\right\|_{L_{w(\alpha)}^{p}} .
$$

(b) Let $\left\{m_{k}\right\}$ be a scalar valued sequence with $m_{0}=0$. Then we have for $\alpha>-1$ and $1 \leq p<\infty$ that

$$
\left\|\left\{m_{k+1}\right\}\right\|_{M_{\alpha ; \alpha}^{p}} \leq C\left\|\left\{m_{k}\right\}\right\|_{M_{\alpha ; \alpha}^{p}} .
$$

Using duality, (b) directly follows from (a), the latter being clear for $p=2$ by Parseval's formula; thus, by the Riesz interpolation theorem, we have only to show (a) in the case $p=1$. By $[18,(5.1 .14)]$ there holds

$$
-\frac{x}{k+1} L_{k}^{\alpha+1}(x)=L_{k+1}^{\alpha}(x)-\left(1+\frac{\alpha}{k+1}\right) L_{k}^{\alpha}(x)
$$

and thus

$$
\left\|\sum_{k=0}^{\infty} a_{k} L_{k+1}^{\alpha}\right\|_{L_{w(\alpha)}^{1}} \leq C\left\|\sum_{k=0}^{\infty} a_{k} L_{k}^{\alpha}\right\|_{L_{w(\alpha)}^{1}}+\left\|\sum_{k=0}^{\infty} \frac{a_{k}}{k+1} L_{k}^{\alpha+1}\right\|_{L_{w(\alpha+1)}^{1}} .
$$

But by $[2,(3.30)]$ and an interchange of the integration order the last term turns out to be dominated by a constant times $\left\|\sum a_{k} L_{k}^{\alpha}\right\|_{L_{w(\alpha)}^{1}}$.

For the converse first note that by $[4,10.12(5)]$

$$
k ! x^{\alpha} e^{-x} L_{k}^{\alpha}(x)=\left((d / d x)^{k-1} e^{-x} x^{k-1+(\alpha+1)}\right)^{\prime}=\left((k-1) ! x^{\alpha+1} e^{-x} L_{k-1}^{\alpha+1}(x)\right)^{\prime}
$$


and, therefore, since $x^{\alpha+1} e^{-x} L_{k-1}^{\alpha+1}(x) \rightarrow 0$ for $x \rightarrow \infty$,

$$
k \int_{x}^{\infty} y^{\alpha} e^{-y} L_{k}^{\alpha}(y) d y=-x^{\alpha+1} e^{-x} L_{k-1}^{\alpha+1}(x) .
$$

Now we estimate the left-shift operator. By (9) and the triangle inequality

$$
\begin{aligned}
\left\|\sum a_{k} L_{k-1}^{\alpha}\right\|_{L_{w(\alpha)}^{1}} & \leq\left\|\sum \frac{k}{k+\alpha} a_{k} L_{k}^{\alpha}\right\|_{L_{w(\alpha)}^{1}} \\
+ & \left\|\sum \frac{a_{k}}{k+\alpha} L_{k-1}^{\alpha+1}\right\|_{L_{w(\alpha+1)}^{1}} \leq C\left\|\sum a_{k} L_{k}^{\alpha}\right\|_{L_{w(\alpha)}^{1}}
\end{aligned}
$$

since $\left\{k_{i} /(k+\alpha)\right\} \in M_{\alpha ; \alpha}^{1}$ and the term with $L_{k-1}^{\alpha+1}$ can be estimated with the aid of (10) and an interchange of the integration order.

Proposition 3.3 allows us to reformulate Corollary 3.1 by iterating the procedure $N$-times, $N \in \mathbf{N}$ fixed, to obtain:

Corollary 3.4 Let $1 \leq p<2, N \in \mathrm{N}$, and $\beta=\alpha-\frac{N p}{2-p}$. Then

$$
\|m\|_{M_{\alpha ; \alpha}^{p}} \leq C \sum_{j=0}^{N}\left\|\left\{(k+1)^{j} \Delta^{j} m_{k}\right\}\right\|_{M_{\beta ; \beta}^{p}}
$$

provided that $\beta>-1$ and $(2 \beta+2)\left(\frac{1}{p}-\frac{1}{2}\right)<1$ when $1<p<2$, and $\beta \geq 0$ when $p=1$.

4. Necessary conditions based on backward differences. Here we want to indicate how backward differences can be used to deduce necessary multiplier criteria and how the transplantation theorem leads to improvements. Starting with (1) we have for $\lambda \geq 0$ that

$$
C x^{\lambda} L_{k}^{\lambda}(x)=L_{k}^{\lambda}(0) \Delta^{\lambda} L_{k}^{0}(x), \quad x>0
$$

and hence for a finite sequence $\left\{a_{k}\right\}$

$$
x^{\lambda} f(x):=x^{\lambda} \sum a_{k} L_{k}^{\lambda}(x)=C \sum a_{k} L_{k}^{\lambda}(0) \Delta^{\lambda} R_{k}^{0}(x)=C \sum \nabla^{\lambda}\left(a_{k} L_{k}^{\lambda}(0)\right) L_{k}^{0}(x)
$$

or by the uniqueness property for Laguerre expansions

$$
\left[x^{\lambda} f(x)\right]_{0}^{\wedge}(k)=C \nabla^{\lambda}\left(a_{k} L_{k}^{\lambda}(0)\right) .
$$

Hölder's inequality gives, with a parameter $\delta \geq 0$ to be chosen later,

$$
\left|\nabla^{\lambda}\left(a_{k} L_{k}^{\lambda}(0)\right)\right| \leq C\|f\|_{L_{w(\lambda-\delta)}^{p}}\left(\int_{0}^{\infty}\left|R_{k}^{0}(x) e^{-x / 2}\right|^{p^{\prime}} x^{\lambda+\delta p^{\prime} / p} d x\right)^{1 / p^{\prime}} .
$$

When estimating the last integral let us restrict to the case $1 \leq p<4 / 3$. Markett's [13] Lemma 1, 5th case, leads to the estimate

$$
\sup _{k}\left|(k+1)^{\delta+(\lambda-\delta+1) / p^{\prime}} \nabla^{\lambda}\left(a_{k} L_{k}^{\lambda}(0)\right)\right| \leq C\|f\|_{L_{w(\lambda-\delta)}^{p}}, \quad \delta<\frac{p}{6}, \lambda \leq-\frac{\delta p^{\prime}}{p}+\frac{p^{\prime}}{6}-\frac{2}{3} .
$$


This inequality is very suited to derive a necessary multiplier criterion in a trivial way (in contrast to the procedure in [5]). As in $[5, I,(9)]$, choose the test function $f=\Phi^{(N)}$, where the Laguerre coefficients of $\Phi^{(N)}$ are smooth, $=1$ if $k \leq N$ and $=0$ if $k \geq 2 N$. There holds [5, I,(9)]

$$
\left\|\Phi^{(N)}\right\|_{L_{w(\lambda-\delta)}^{p}} \leq C(N+1)^{(\alpha+1) / p^{\prime}+\delta / p}
$$

and hence, with $a_{k}=m_{k}\left[\Phi^{(N)}\right]_{\lambda}^{\wedge}(k)$ and $m \in M_{\lambda ; \lambda-\delta}^{p}$, the following result via (5).

Corollary 4.1 Let $0 \leq \lambda \leq-\frac{\delta p^{\prime}}{p}+\frac{p^{\prime}}{6}-\frac{2}{3}, 0 \leq \delta<\frac{p}{6}, \beta=\lambda-\frac{2 \delta}{2-p}$, and let $\lambda, \beta$ satisfy the first two conditions of (6). Then

$$
\sup _{k}\left|\nabla^{\lambda}\left(m_{k} L_{k}^{\lambda}(0)\right)\right| \leq C\|m\|_{M_{\lambda ; \lambda-\delta}^{p}} \leq C\|m\|_{M_{\beta ; \beta}^{p}}, \quad 1<p<4 / 3 .
$$

REMARK 4. The use of the parameter $\delta$ (near $p / 6)$ and the resulting application of the transplantation theorem (5) yields a definite improvement of a necessary condition resulting from the choice $\delta=0$ (when no transplantation is needed). Consider e.g. the case $p=7 / 6,0 \leq \delta \leq 1 / 18$ which implies at least $\lambda \leq 1 / 6$. Take as test multiplier on $L_{w(0)}^{p}$ the one corresponding to the partial sum operator, i.e., $m_{k}^{(n)}=1,0 \leq k \leq n$, and $=0$ otherwise.

Choosing $\delta=0, \lambda=\beta=0$, only gives $O(1)$ as lower bound whereas $\delta=1 / 18, \beta=$ 0 leads to the admissible $\lambda=2 / 15$ and a divergence behavior of the same multiplier family on the same $L^{p}$-space of at least $O\left((n+1)^{2 / 15}\right)$. Corollary 1.1 in $[5, \mathrm{I}]$, which describes a necessary condition based on forward differences with increment 1 , gives for this example the slightly better result $O\left((n+1)^{1 / 7}\right)$ (with the present backward differences one can still try to optimize $\delta<p / 6)$. There it is also shown that for $p=1[5$, Corollary 1.1] cannot be improved though it does not yield the correct divergence behavior of the partial sum operator. In $[5, \mathrm{II}]$ it is shown that via the use of differences with mixed increment 1 and 2 one can obtain the "right" divergence behavior $O\left((n+1)^{3 / 14}\right)$.

The example just considered again makes clear the need for a transplantation theorem with full range power weights.

5. The value of Corollary 4.1 is more to be seen in the fact that it allows one to integrate the Kalnel-type necessary conditions, which looked a bit isolated in the framework of those conditions known till now (see [5] and [16]). In [8] the following sharp criterion is shown:

Let $\alpha \geq 0$ and $m=\left\{m_{k}\right\}$ be a finite sequence with $m_{k}=0$ for $k \geq n+1$. Then

$$
\sum_{k=0}^{n}\left|m_{k}\right| \frac{(k+1)^{\alpha+1 / 2}}{(n+1-k)^{\alpha+3 / 2}} \leq C\|m\|_{M_{\alpha ; \alpha}^{1}} .
$$

Intuitively it is clear that the left hand side is an upper bound for $\nabla^{\alpha+1 / 2}\left(m_{n} L_{n}^{\alpha+1 / 2}(0)\right)$, the type of condition occurring in Corollary 4.1. Unfortunately it is not clear to the authors why the difference order is $1 / 2$ higher than in Corollary 4.1 . 


\section{REFERENCES}

[1] K. F. ANDERSEN AND H. P. HeINIG, Weighted norm inequalities for certain integral operators, SIAM J. Math. Anal., 14 (1983), pp. 834-844.

[2] R. ASKEY AND J. FITCH, Integral representations for Jacobi polynomials and some applications, J. Math. Anal. Appl., 26 (1969), pp. 411-437.

[3] R. R. Coifman And G. Weiss, Transference Methods is Analysis, CBMS 31, Amer. Math. Soc., Providence, R. I., 1977.

[4] A. Erdelyi, W. Magnus, F. Oberhettinger, and F. G. Tricomi, Higher Transcendental Functions, I \& II, McGraw-Hill, New York, 1953.

[5] G. GASPER AND W. TREBels, On necessary multiplier conditions for Laguerre expansions, Canad. J. Math., 43 (1991), pp. 1228-1242; II, SIAM J. Math. Anal., 25 (1994), pp. 384391.

[6] G. Gasper AND W. TRebels, On a restriction problem of de Leeuw type for Laguerre multipliers, Acta Math. Hungar., 68 (1995), pp. 135-149.

[7] G. Gasper and W. TRebels, Ultraspherical multipliers revisited, Acta Sci. Math. (Szeged), 60 (1995), pp. 291-309.

[8] G. Gasper AND W. TREBels, A lower estimate for the Lebesgue constants of linear means of Laguerre expansions, Results Math., 34 (1998), pp. 91-100.

[9] E. Görlich AND C. MARKetT, A convolution structure for Laguerre series, Indag. Math., 44 (1982), pp. 161-171.

[10] D. L. GuY, Hankel multiplier transformations and weighted p-norms, Trans. Amer. Math. Soc., 95 (1960), pp. 137-189.

[11] S. G. KAL'NEI, On a lower estimate of the Lebesgue function of linear means of Fourier-Jacobi series, Proc. Steklov Inst. Math., 170 (1987), pp. 127-133.

[12] Y. Kanjin, A transplantation theorem for Laguerre series, Tôhoku Math. J., 43 (1991), pp. 537-555.

[13] C. MARKETT, Mean Cesàro summability of Laguerre expansions and norm estimates with shifted parameter, Anal. Math., 8 (1982), pp. 19-37.

[14] B. Muckenhoupt, Transplantation Theorems and Multiplier Theorems for Jacobi Series, Memoirs Amer. Math. Soc., no. 356, vol. 64, Amer. Math. Soc., Providence, R.I., 1986.

[15] E. L. PoIAnI, Mean Cesàro summability of Laguerre and Hermite series, Trans. Amer. Math. Soc., 173 (1972), pp. 1-31.

[16] K. STEMPAK AND W. TREBels, On weighted transplantation and multipliers for Laguerre expansions, Math. Ann., 300 (1994), pp. 203-219.

[17] S. Thangavelu, Lectures on Hermite and Laguerre expansions, Mathematical Notes 42, Princeton Univ. Press, Princeton, NJ, 1993.

[18] G. Szegö, Orthogonal Polynomials (4th ed.), Amer. Math. Soc. Colloq. Publ. 23, Providence, R.I., 1975.

[19] M. ZAFRAN, Interpolation of multiplier spaces, Amer. J. Math., 105 (1983), pp. 1405-1416. 